\title{
Two-year follow-up of low-level laser therapy for elderly with painful adhesive capsulitis of the shoulder
}

This article was published in the following Dove Press journal: Journal of Pain Research

25 May 2015

Number of times this article has been viewed

\section{David IP \\ Nga-Yue Fu}

Asia Medical Pain Centre, Mong Kok, Hong Kong,

People's Republic of China
Correspondence: David Ip Asia Medical Pain Centre, 4/F Tower 2 Grand Plaza, Mong Kok, Hong Kong, People's Republic of China Tel +852 23880389

Email ipdavid@hotmail.com.hk
Introduction: This paper reports on the medium-term mean 2-year prospective follow-up of a patient cohort of 35 unselected elderly patients with mean age of 65 years who visited our tertiary referral pain center for painful adhesive capsulitis of the shoulder managed with lowlevel laser therapy (LLLT).

Materials and methods: All patients in this prospective cohort study had documentation of the diagnosis by contrast-enhanced magnetic resonance imaging before study entry and all had failed to respond to a combination of conventional physical therapy and nonsteroidal anti-inflammatory medications for not fewer than 4 weeks. LLLT, at a wavelength of $810 \mathrm{~nm}$ emitted from a GaAIAs semiconductor laser device with $5.4 \mathrm{~J}$ per point and a power density of $20 \mathrm{~mW} / \mathrm{cm}^{2}$, was employed to irradiate six predetermined anatomic points and two acupuncture points. The treatment regimen consisted of three sessions of treatment per week for 8 consecutive weeks. Each treatment session lasted 180 seconds. Serial clinical assessment was undertaken using the Constant-Murley shoulder score.

Results: A total of 50 painful shoulder joints were treated, as a number of elderly presented with bilateral symptoms. All but four painful shoulders showed significant improvement in Constant-Murley shoulder score at the end of 8-weeks' LLLT treatment and, surprisingly, the improvement was found maintained at follow-up assessments at 1 year and 2 years.

Conclusion: We conclude that LLLT is a viable option in the conservative treatment of shoulder pain arising from adhesive capsulitis of the shoulder in the elderly, with a positive clinical result of more than $90 \%$ and with clinical efficacy both in the short-term and the medium-term.

Keywords: Constant-Murley shoulder score, LLLT, shoulder pain, acupuncture point

\section{Introduction}

The significance of low-level laser in clinical medicine began with the important works of Endre Mester. The basic science underlying low-level laser therapy (LLLT) is that it involves the treatment of various medical diseases with photomedicine and the use of low-level lasers or light-emitting diodes to alter cellular function, or, in short, the employment of photo-biomodulation effects to help normalize cellular functions, including the energy usage of body cells.

In recent years, the various clinical applications of LLLT have mainly been based on previous scientific works concerning the effect of low-level lasers, such as increasing adenosine triphosphate production, ${ }^{1}$ while, at the same time, the exertion of a positive influence on fibroblast ${ }^{2}$ and collagen synthesis ${ }^{3}$ at the cellular-molecular level. Clinical protocols exist for LLLT in the management of common painful conditions such as rheumatoid arthritis, osteoarthritis, acute and chronic neck pain, tendinopathy, and chronic joint disorders. 
As far as adhesive capsulitis of the shoulder in the elderly is concerned, most clinicians use a combination of nonsteroidal anti-inflammatory medications and conventional physical therapy consisting of ultrasonic therapy, transcutaneous electrical therapy, and short-wave therapy, for management. These forms of conservative treatment represent symptomatic treatment only, without the biomodulation effects offered by low-level lasers; for instance, ultrasound treatment affords neither anti-inflammatory nor biomodulation effects at the cellular-molecular level. This is in sharp contrast to LLLT, which confers the mentioned biomodulation effects.

The objective of this paper is to report the clinical result of a study on the efficacy of LLLT in the management of the early phase of symptomatic adhesive capsulitis of the shoulder in elderly who failed to respond to the combined use of conventional physical therapy and nonsteroidal antiinflammatory medications for not fewer than 4 weeks. In this study, we investigated the clinical effect of the combined irradiation of strategic anatomic points of pathology in adhesive capsulitis as well as acupuncture points, which, to the best of our knowledge, has not been reported in the literature to date.

We stress that patients presenting late, after 6 weeks of disease onset, were excluded to guard against the possibility that whatever positive clinical result obtained from the use of LLLT could have been due to spontaneous disease resolution.

Published medical literature exists concerning clinical studies of the effect of LLLT on frozen shoulder that were positive, albeit being short-lived. ${ }^{4-6}$ Prior to the start of the present study, we researched the irradiation of two acupuncture points - namely, Bingfeng and Tianzong - in addition to the laser irradiation, at a wavelength of $810 \mathrm{~nm}$, of six preselected points that are commonly abnormal on magnetic resonance imaging studies in patients with adhesive capsulitis. We found long-lasting clinical results, particularly with regard to improvement in range of motion; much longer, in fact, than that previously reported in the literature. This forms the rationale of this clinical study and forms the rationale as to why we followed up the patients for a mean of 2 years.

\section{Materials and methods}

The study population consisted of a prospective cohort of consecutive unselected 35 elderly patients with mean age of 65 (range: 60-77) years being referred to our tertiary referral pain center within 6 weeks of onset of symptoms and previously having failed response to a combination of nonsteroidal anti-inflammatory medications and not fewer than 4 weeks of conventional physical therapy. Contrast-enhanced magnetic resonance imaging was performed in each patient prior to entry to the study to confirm the typical appearances in the axillary pouch, rotator interval, biceps anchor, and anteroposterior capsule ${ }^{7}$ to confirm the diagnosis and ensure there was no other concomitant pathology such as rotator-cuff tear. Exclusion criteria included patients presenting late after 6 weeks of disease onset; patients who had concomitant other shoulder pathologies on magnetic resonance imaging; patients with prior shoulder operations; and patients who had potential contraindications for the use of laser treatment, such as previous history of tumor or ongoing sepsis. We also excluded patients with previous neuromuscular conditions of the affected upper extremity, such as a previous cerebrovascular accident. All patients signed informed consent detailing that they would be treated by LLLT and that only US Food and Drug Administration-approved devices would be used.

Each subject was clinically examined and followed up by the same orthopedic surgeon to minimize interobserver error. All the low-level laser pain treatments were also performed by the same orthopedic surgeon, and all the serial shoulder scoring was done by the patient with the assistance of the same orthopedic surgeon. During the initial visit, extra care was taken to document the shoulder range of motion, presence of neurological deficit, sites of tenderness, and overall upper extremity function. We employed the well-known Constant-Murley score ${ }^{8}$ a 100 -point scale composed of a number of individual parameters, to assess the patient's level of pain and ability to carry out normal daily activities. The test is divided into four subscales: pain (15 points); activities of daily living ( 20 points); strength (25 points); and range of motion: forward elevation, external rotation, abduction, and internal rotation of the shoulder (40 points) - the higher the score, the higher the quality of the function.

LLLT - at a wavelength of $810 \mathrm{~nm}$ emitted from a GaAIAs semiconductor laser device (Figure 1) with 5.4 J per point, $32.4 \mathrm{~J}$ per session, spot area of $0.5 \mathrm{~cm}^{2}$, peak power of $50 \mathrm{~mW}$, and power density of $20 \mathrm{~mW} / \mathrm{cm}^{2}$, and continuous output - was employed. The treatment regimen consisted of three sessions of treatment per week for 8 consecutive weeks. Each treatment session lasted 180 seconds: 20 seconds each for the six chosen spots - such as, the subacromial space, biceps anchor, axillary pouch, anterior shoulder capsule, posterior shoulder capsule, and rotator interval. In addition, we irradiated two acupuncture points ${ }^{9,10}$ near the shoulder for 30 seconds each - the Tianzong and Bingfeng acupoints (Figure 2), otherwise known as SI 11 and SI 12, respectively, of 


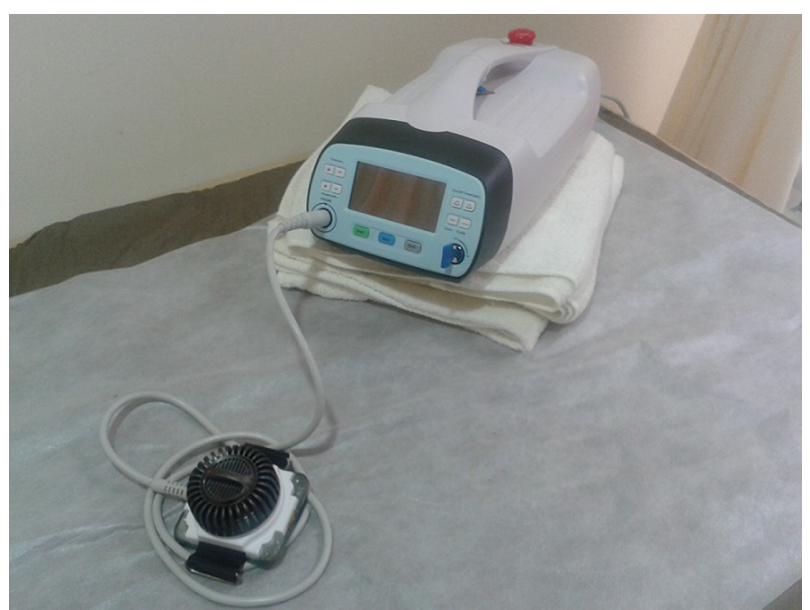

Figure I Laser device ( $810 \mathrm{~nm}$ wavelength) used in irradiation of six anatomically relevant points as well as two acupuncture points, Bingfeng and Tianzong.

the small intestine meridian in the field of Chinese medicine. Protective laser eye goggles were provided to both the patient and the clinician. We did not add on any other conventional therapy during each treatment session, as all the subjects had had these therapeutic interventions before but had failed to respond. Further, no medications were prescribed to the subjects under study, as none of the patients had responded to oral anti-inflammatory agents provided by other clinicians before receiving treatment at our tertiary referral pain center. The only rehabilitation exercises were the home exercises already taught to patients by other physiotherapy centers prior to patients' entry to the study. Therefore, essentially all

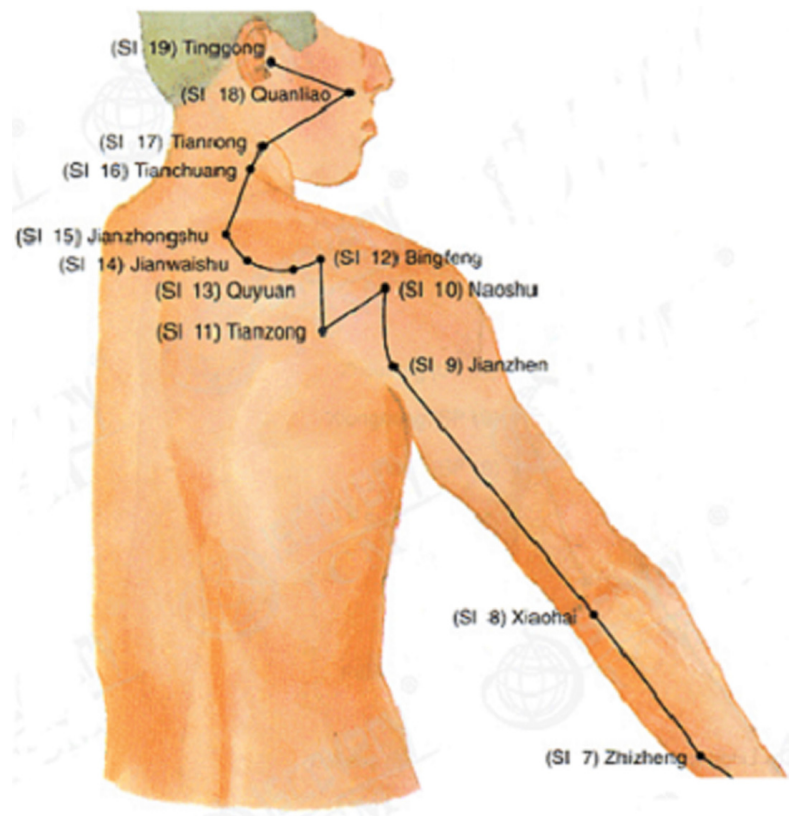

Figure 2 Picture showing the location of the Bingfeng and Tianzong acupuncture points near the shoulder. subjects in the current study received only LLLT as really the sole therapy. Figure 3 shows a patient in receipt of irradiation of an acupuncture point.

\section{Results}

In this study of 35 elderly patients and 50 painful and stiff shoulders affected by painful adhesive capsulitis, the male to female ratio was 1.0:1.3 and the right shoulder was affected in $60 \%$ of cases. The study period lasted from 2011 to the end of 2014.

All 35 subjects in the study population completed the treatment regimen with good compliance; there were no defaults. No side effect or any patient discomfort was documented during all treatments. Only four painful shoulders among the 50 shoulders failed to positively respond to laser treatment, with no improvement in Constant-Murley score, and eventually required arthroscopic surgery.

Table 1 details the relevant demographics of the patient population together with the serial pre- and posttreatment shoulder scores. Figure 4 shows the relative breakdown of the mean value of the constant subscores pre- and posttreatment as well as at 2-year follow-up, including the range of motion subscale. In the graph, one can notice that LLLT treatment had a positive clinical effect on range of motion, level of pain, and activities of daily living but had no effect on the strength of the elderly patients.

Apart from the four shoulders of two patients who did not show clinical response to LLLT and required surgery, there was a statistically significant improvement in the constant score in the remaining 46 shoulders, from a mean score of 59 at the start of the study (range: 57-62), with standard deviation of 1.1 to a mean score of 71 at the end of treatment

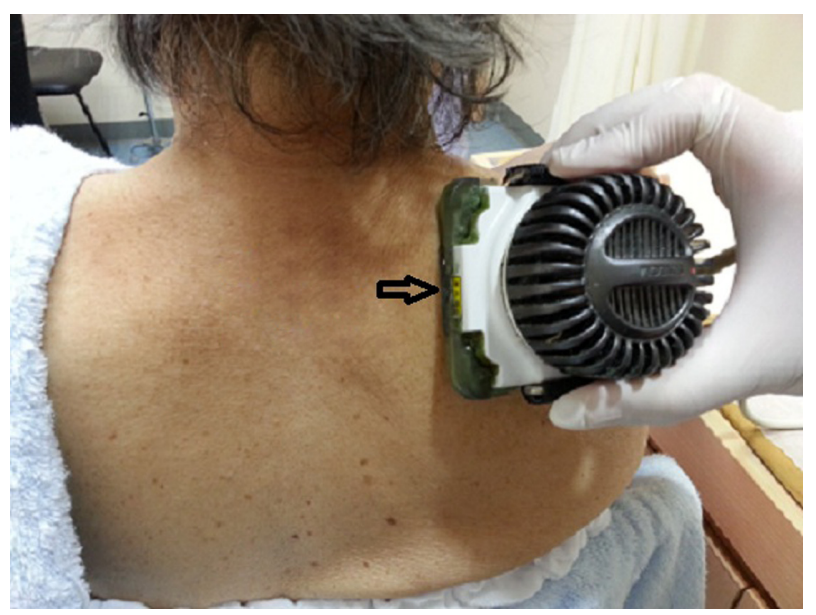

Figure 3 Laser device ( $810 \mathrm{~nm}$ wavelength; indicated by the arrow) irradiating the acupuncture point. 
Table I Demographics of the patient population together with the serial pre- and posttreatment shoulder scores

\begin{tabular}{|c|c|c|c|c|}
\hline \multirow{2}{*}{$\begin{array}{l}\text { Patient (sex, } \\
\text { age [years]) }\end{array}$} & \multirow{2}{*}{$\begin{array}{l}\text { Shoulder } \\
\text { affected }\end{array}$} & \multicolumn{3}{|l|}{ Score } \\
\hline & & Pre-Rx & Post-Rx & At 2-year follow-up \\
\hline Female, 64 & Right & 61 & 72 & 72 \\
\hline \multirow[t]{2}{*}{ Male, 73} & Right & 57 & 71 & 70 \\
\hline & Left & 58 & 72 & 72 \\
\hline Female, 68 & Left & 60 & 70 & 69 \\
\hline \multirow[t]{2}{*}{ Male, 77} & Right & 57 & 71 & 70 \\
\hline & Left & 57 & 71 & 70 \\
\hline Female, 66 & Left & 60 & 70 & 70 \\
\hline \multirow[t]{2}{*}{ Female, 66} & Right & 59 & 69 & 69 \\
\hline & Left & 59 & 70 & 69 \\
\hline Male, 60 & Right & 62 & 73 & 73 \\
\hline \multirow[t]{2}{*}{ Female, 62} & Right & 59 & 73 & 72 \\
\hline & Left & 60 & 70 & 70 \\
\hline Male, 6I & Right & 61 & 73 & 73 \\
\hline \multirow[t]{2}{*}{ Female, 66} & Right & 58 & 72 & 72 \\
\hline & Left & 59 & 69 & 69 \\
\hline Male, 65 & Right & 61 & 71 & 70 \\
\hline \multirow[t]{2}{*}{ Female, 70} & Right & 57 & 57 & Surgery done \\
\hline & Left & 57 & 57 & Surgery done \\
\hline Female, 66 & Right & 60 & 70 & 70 \\
\hline Male, 67 & Left & 61 & 71 & 71 \\
\hline \multirow[t]{2}{*}{ Female, 69} & Right & 57 & 73 & 72 \\
\hline & Left & 58 & 72 & 72 \\
\hline Male, 65 & Right & 62 & 73 & 72 \\
\hline \multirow[t]{2}{*}{ Female, 65} & Right & 60 & 70 & 70 \\
\hline & Left & 58 & 68 & 68 \\
\hline \multirow[t]{2}{*}{ Female, 62} & Right & 60 & 71 & 71 \\
\hline & Left & 60 & 71 & 71 \\
\hline Male, 63 & Right & 59 & 69 & 69 \\
\hline Female, 6I & Right & 62 & 71 & 72 \\
\hline \multirow[t]{2}{*}{ Male, 64} & Right & 60 & 70 & 70 \\
\hline & Left & 59 & 69 & 69 \\
\hline Male, 62 & Right & 61 & 71 & 70 \\
\hline \multirow[t]{2}{*}{ Female, 65} & Right & 59 & 69 & 69 \\
\hline & Left & 59 & 70 & 69 \\
\hline Female, 66 & Right & 57 & 72 & 71 \\
\hline \multirow[t]{2}{*}{ Female, 68} & Right & 57 & 73 & 72 \\
\hline & Left & 58 & 72 & 72 \\
\hline \multirow[t]{2}{*}{ Male, 72} & Right & 57 & 57 & Surgery done \\
\hline & Left & 58 & 58 & Surgery done \\
\hline Female, 67 & Right & 58 & 72 & 72 \\
\hline Male, 70 & Right & 57 & 72 & 70 \\
\hline \multirow[t]{2}{*}{ Female, 68} & Right & 58 & 70 & 70 \\
\hline & Left & 60 & 72 & 72 \\
\hline Male, 6I & Left & 58 & 70 & 70 \\
\hline Female, 60 & Right & 59 & 69 & 69 \\
\hline \multirow[t]{2}{*}{ Male, 63} & Right & 57 & 72 & 72 \\
\hline & Left & 58 & 73 & 72 \\
\hline Female, 60 & Right & 60 & 70 & 70 \\
\hline Male, 6I & Right & 59 & 69 & 69 \\
\hline Female, 60 & Left & 61 & 72 & 71 \\
\hline
\end{tabular}

Abbreviation: $\mathrm{RX}$, prescription.

(range: 68-73), with standard deviation of 1.0. Statistical testing with Student's $t$-test rejected the null hypothesis that the consistent improvement in Constant-Murley shoulder scores could have arisen by chance, at $P<0.05$.
The mean clinical follow-up of this patient cohort was 2 years (range: $1.5-2.5$ years). A reasonably long follow-up was required to reveal whether the LLLT modality alone could provide any lasting benefits in patients, as well as any incipient side effects. In this study, no side effect was observed at the 2-year mark, and all patients tolerated the cold laser therapy very well.

\section{Discussion}

Over the past years, more than 100 double-blind placebocontrolled studies have been published on the effects of LLLT. These articles also showed the favorable anti-inflammatory effect of LLLT. ${ }^{11}$ Low-level lasers may reduce pain related to inflammation by lowering, in a dose-dependent manner, levels of prostaglandin E2, prostaglandin-endoperoxide synthase 2, interleukin 1-beta, tumor necrosis factor-alpha, the cellular influx of neutrophil granulocytes, oxidative stress, and tissue edema. The appropriate dose appears to be between 0.3 and $19.0 \mathrm{~J} / \mathrm{cm}^{2} .{ }^{11}$

Unlike traditional laser devices, innumerous clinical studies have shown that low-level lasers are safe and lack side effects, unlike, for example, the lasers used for cosmetic surgery, in which thermal injury to the skin is not an uncommon side effect. The current study employed an $810 \mathrm{~nm}$ wavelength laser and we irradiated six important anatomic points around the shoulder region relevant to the shoulder pathology at hand. Additionally, we irradiated the Bingfeng and Tianzong acupuncture points to produce additive effects, especially range of motion improvement, since previous studies of LLLT on adhesive capsulitis did not consistently achieve an improved range of motion despite achieving pain relief. ${ }^{2}$ The total application time of all irradiation, 180 seconds, was well tolerated, even by patients of advanced age. There was absolutely no need for any anesthesia of any kind, since the laser application was noninvasive and painless.

The majority of previous clinical studies of LLLT concentrated more on shoulder impingement syndrome ${ }^{12}$ than on frozen shoulder, but the reported positive effects appeared only short-lived, ${ }^{13}$ as claimed by a recent Cochrane systematic review, and there is a complete lack of medium-term clinical follow-up studies on the subject in the medical literature. To add to this, studies that have investigated shoulder pain in general, without pinpointing a particular diagnosis, have, in fact, looked at an admixture of different shoulder disorders, therefore it is precarious to draw any conclusions from them. For instance, in many clinical studies, the researchers relied solely on a clinical diagnosis without confirmation by magnetic resonance imaging. Magnetic resonance imaging 


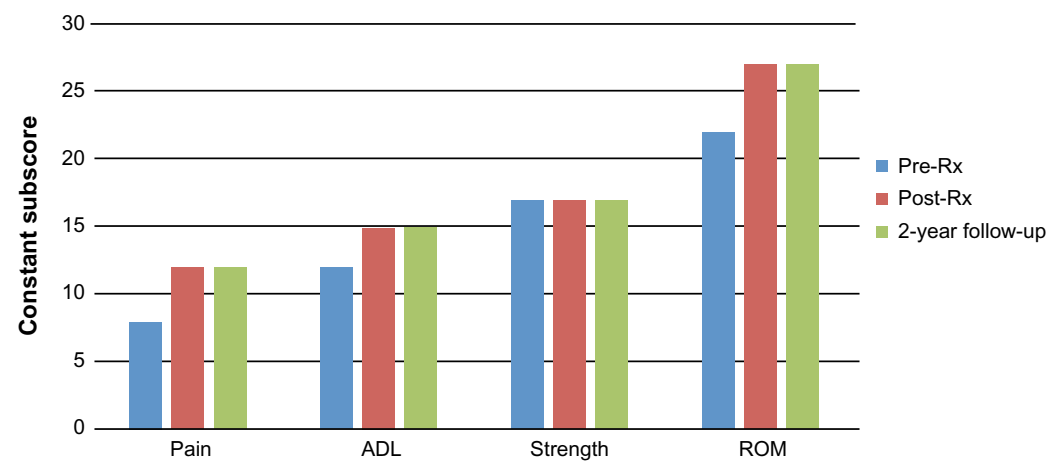

Figure 4 Mean pre- vs post-prescription (Rx) constant subscores.

Abbreviations: ADL, activities of daily living; ROM, range of motion.

is imperative to rule out other possible shoulder pathologies, besides confirming the clinical diagnosis beyond an element of doubt.

In summary, we chose LLLT owing to its lack of side effects, while being noninvasive and well tolerated by the elderly. By contrast, traditional methods of treatment for painful shoulders of the elderly caused by adhesive capsulitis involve nonsteroidal anti-inflammatory agents as well as conventional physiotherapy, such as ultrasound therapy and electrical stimulation therapy. None of these therapies has the biomodulation effects offered by LLLT, such as improvement in microcirculation or the upregulation of several genes involved in energy metabolism and oxidative phosphorylation, which stimulates an increase in adenosine triphosphate production, which in turn regulates other cellular processes, leading to the normalization of biological functions at the cellular level. For instance, therapeutic ultrasound devices do not even have anti-inflammatory effects let alone any biomodulation effects. ${ }^{14}$

In general, for any conservative treatment of adhesive capsulitis to be meaningful, the treatment modality must have lasting effects and benefits for the patient, rather than providing only transient pain relief, such as the administration of painkillers does. We are not aware of any clinical study reporting on the medium-term efficacy of LLLT in the management of shoulder adhesive capsulitis. In the study reported here, LLLT showed evidence of lasting benefit and represents a viable option to choose rather than surgery, particularly in the elderly population, in whom concomitant medical comorbidities may well increase the risk of surgery.

\section{Limitations and future directions}

This study lacked a control group, which represents a weakness and is acknowledged as such. A control group was not included, as the majority of patients do not give consent to the idea of a sham laser light source. The fact that the patient population included subjects who failed to respond to a combination of conventional physical therapy and nonsteroidal anti-inflammatory medications but responded positively to LLLT warrants further large-scale double-blind placebo-controlled studies to further confirm the clinical results of this study, provided lasers of similar wavelength, power density, and application time, and the six points of application are the same, together with the two additional acupuncture points of Bingfeng and Tianzong to make any clinical results comparable.

\section{Conclusion}

The current prospective review of a patient cohort of 35 elderly patients with documented symptomatic adhesive capsulitis of 50 painful shoulders showed a significant positive clinical response in patients, as evidenced by the marked improvement in their Constant-Murley scores, not only in the short-term but also in the medium-term, as the mean clinical follow-up in this prospective study was 2 years.

Further large-scale studies are worthwhile to further explore the use of LLLT, not only in the treatment of adhesive capsulitis but also in other painful shoulder conditions, such as partial rotator-cuff tears.

\section{Disclosure}

The authors declare no conflicts of interest in this work.

\section{References}

1. Hashmi JT, Huang YY, Osmani BZ, Sharma SK, Naeser MA, Hamblin MR. Role of low-level laser therapy in neurorehabilitation. PM R. 2010; 2(12 Suppl 2):S292-S305.

2. van Breugel HH, Bär PR. Power density and exposure time of $\mathrm{He}-\mathrm{Ne}$ laser irradiation are more important than total energy dose in photobiomodulation of human fibroblasts in vitro. Lasers Surg Med. 1992;12(5):528-537.

3. Lam TS, Abergel RP, Meeker CA, Castel JC, Dwyer RM, Uitto J. Laser stimulation of collagen synthesis in human skin fibroblast cultures. Lasers Life Sci. 1986;1:61-77. 
4. Stergioulas A. Low-power laser treatment in patients with frozen shoulder: preliminary results. Photomed Laser Surg. 2008;26(2):99-105.

5. Jain TK, Sharma NK. The effectiveness of physiotherapeutic interventions in treatment of frozen shoulder/adhesive capsulitis: a systematic review. J Back Musculoskelet Rehabil. 2014;27(3):247-273.

6. Soliman AS, Mahmoud AM, Serry ZM, Dawood FG. Therapeutic effects of low-level laser and reflexology on adhesive capsulitis in elderly type 2 diabetic patients. Asian J Pharm Clin Res. 2014;7(5):317-321.

7. Gokalp G, Algin O, Yildirim N, Yazici Z. Adhesive capsulitis: contrast-enhanced shoulder MRI findings. J Med Imaging Radiat Oncol. 2011;55(2):119-125.

8. Constant CR, Murley AH. A clinical method of functional assessment of the shoulder. Clin Orthop Relat Res. 1987;(214):160-164.

9. Jin D, Li Z. Acupuncture and the elevation manipulation of massage for treatment of frozen shoulder. J Tradit Chin Med. 2003;23(3):212-213.
10. Liu H, Zhang C. 60 cases of shoulder-arm syndrome treated by electroacupuncture at Bingfeng (SI 12). J Tradit Chin Med. 1998;18(4): 256-258.

11. Bjordal JM, Johnson MI, Iversen V, Aimbire F, Lopes-Martins RA. Low-level laser therapy in acute pain: a systematic review of possible mechanisms of action and clinical effects in randomized placebocontrolled trials. Photomed Laser Surg. 2006;24(2):158-168.

12. Kelle B, Kozanoglu E. Low-level laser and local corticosteroid injection in the treatment of subacromial impingement syndrome: a controlled clinical trial. Clin Rehabil. 2014;28(8):762-771.

13. Page MJ, Green S, Kramer S, Johnston RV, McBain B, Buchbinder R. Electrotherapy modalities for adhesive capsulitis (frozen shoulder). Cochrane Database Syst Rev. 2014;10:CD011324.

14. Baker KG, Robertson VJ, Duck FA. A review of therapeutic ultrasound: biophysical effects. Phys Ther. 2001;81(7):1351-1358.
Journal of Pain Research

\section{Publish your work in this journal}

The Journal of Pain Research is an international, peer-reviewed, open access, online journal that welcomes laboratory and clinical findings in the fields of pain research and the prevention and management of pain. Original research, reviews, symposium reports, hypothesis formation and commentaries are all considered for publication.

\section{Dovepress}

The manuscript management system is completely online and includes a very quick and fair peer-review system, which is all easy to use. Visit http://www.dovepress.com/testimonials.php to read real quotes from published authors. 